\title{
Biología aplicada: una aproximación interdisciplinar a la investigación del cáncer
}

\author{
Applied Biology: \\ An interdisciplinary approach to the cancer research
}

ANNA ESTANY

Universitat Autònoma de Barcelona (España)

Recibido: 17-1-2013

Aprobado definitivamente: 07-2-2013

\section{RESUMEN}

La importancia de la biología aplicada tiene que ver con una serie de fenómenos en torno al papel de la ciencia y la tecnología en nuestra sociedad. Entre dichos fenómenos podemos señalar la imbricación que en la actualidad existe entre la investigación básica, la utilización de ésta por las ciencias de diseño y la construcción tecnología para cambiar la realidad. El objetivo de este trabajo es analizar el papel de la biología molecular en la investigación del cáncer, a partir del marco teórico de las ciencias de diseño de H. Simon (1969), la reflexión filosófica de I. Niiniluoto (1993), y la praxiología como ciencia de la acción eficiente de T. Kotarbinski (1965).

PALABRAS CLAVE

CIENCIA APLICADA, INVESTIGACIÓN BÁSICA, CIENCIAS DE DISEÑO, INVESTIGACIÓN SOBRE EL CÁNCER

\footnotetext{
* Este trabajo se ha realizado en el marco del proyecto de investigación «Innovación en la práctica científica: aspectos cognitivos y sus consecuencias filosóficas» (FFI 2011-23238), financiado por el Ministerio de Ciencia e Innovación de España.

(C) Contrastes. Revista Internacional de Filosofía: Suplemento 18 (2013), pp. 289-301. ISSN: 1136-9922 Departamento de Filosofía, Universidad de Málaga, Facultad de Filosofía y Letras

Campus de Teatinos, E-29071 Málaga (España)
} 


\section{ABSTRACT}

The importance of applied biology has to do with a series of events around the role of science and technology in our society. The relevant point is the relationship that exists between basic research, the use of this design in science and construction technology to change the reality. The aim of this paper is to analyze the role of molecular biology in cancer research, from the theoretical framework of design science H. Simon (1969), philosophical reflection of I. Niiniluoto (1993), and Praxeology as a science of efficient action of T. Kotarbinski (1965).

KEYWORDS

APPLIED SCIENCE, BASIC RESEARCH, DESIGN SCIENCE, CANCER RESEARCH

\section{INTRODUCCIÓN}

LA RELACIÓN ENTRE CIENCIA PURA Y APLICADA, y entre ciencia y tecnología nunca ha sido una cuestión central en la filosofía de la ciencia clásica, ${ }^{1}$ aunque ha habido aportaciones dignas de mención por parte de algunos filósofos. ${ }^{2}$ En el caso de la biología tampoco ha sido prioritario el análisis de la aplicación de la misma. De todas formas, si entendemos la biología aplicada en sentido amplio, es decir, en abordar el impacto de los resultados de la investigación biológica en la sociedad, podríamos considerar buena parte de la bioética o de la biotecnología como parte del campo de la biología aplicada. Pero en este caso las aportaciones no proceden de la filosofía de la ciencia en sentido estricto.

La importancia de la biología aplicada tiene que ver con una serie de fenómenos en torno al papel de la ciencia y la tecnología en nuestra sociedad. Entre dichos fenómenos podemos señalar la imbricación que en la actualidad existe entre la investigación básica, la utilización de ésta por las ciencias de diseño (ciencias que transforman el mundo, como las ingenierías, la medicina, la biblioteconomía, la didactología, etc.) y la construcción de artefactos (tecnología) para cambiar la realidad. Esto es posible por la rapidez con la que se pasa del conocimiento científico a su aplicación para satisfacer necesidades humanas o construir tecnología. M. Kranzberg, historiador de la tecnología, señala en «The unity of science-technology» (1967) que pasaron 1700 años desde que la máquina de vapor fuera diseñada en Alejandría hasta que Watt

1 Me refiero a los modelos de ciencia predominantes durante la primera mitad del siglo XX y que, en buena parte, corresponden a la denominada «Concepción heredada». Pero incluso en la filosofía de la ciencia de corte historicista de las décadas de los sesenta y setenta los casos históricos estudiados corresponden a ciencia pura.

2 Por ejemplo, el volumen 2 de Philosophy of science, de 1976, sobre filosofía de la tecnología con trabajos de M. Bunge, P.T. Durbin, R. Giere y E.T. Layton, entre otros. 
la hizo funcionar, el principio de fotografía tardó en llevarse a la práctica 200 años desde que fue esquematizado por Leonardo, el motor eléctrico tardó 40 años, la energía nuclear 5 años, el transistor 5 años, los plásticos transparentes 2 años y los rayos láser 18 meses. Esto, dice Kranzberg, apoya la tesis de que la asociación de la ciencia (que quiere saber el «porqué») y la tecnología (que quiere saber el «cómo») produce una reacción en cadena de descubrimiento científico e invención tecnológica. En el caso de la biología podemos pensar, por un lado, en cómo el estudio del genoma humano, la investigación con células madres, etc. tienen consecuencias en la medicina y, por otro, cómo esto puede afectar a decisiones políticas sobre el sistema sanitario, las mutuas aseguradoras, etc. Vemos pues que esta rapidez e interconexión entre conocimiento y aplicación práctica, que señala Kranzberg, tiene en la biología una relevancia capital.

El objetivo de este trabajo es analizar el papel de la biología molecular en la investigación del cáncer en tanto en cuanto constituye ciencia aplicada y, en consecuencia, intervienen factores tanto epistemológicos como sociales. La investigación del cáncer es un caso de biología molecular aplicada en el que inciden diversos factores como resultado de la imbricación ya mencionada entre conocimiento científico, tecnología y consecuencias para la sociedad. A ello hay que añadir que cualquiera de los resultados relacionados con los tumores afecta la vida de miles de personas a nivel planetario y, en consecuencia, agranda la propia discusión científica, poniendo en el centro del debate la relación entre las ciencias puras y aplicadas. Especial atención merece la idea de progreso y cómo éste se entiende en la ciencia aplicada en general y en la investigación del cáncer en particular.

\section{TENSIÓN ENTRE CIENCIA PURA Y CIENCIA APLICADA}

La tensión entre ciencia pura y aplicada tiene especial incidencia en la investigación del cáncer. Para ejemplificar este fenómeno vamos a referirnos a un estudio de un grupo de científicos y filósofos alemanes que en las décadas de los 70 y 80 realizaron sobre las relaciones entre investigación básica y medicina en Alemania a raíz de la investigación del cáncer. R. Hohlfeld (1983) entrevistó a 29 científicos que trabajaban en campos de biología molecular, investigación experimental del cáncer, investigación clínica del cáncer, medicina del cáncer y epidemiología entre los años 1975 y 1976. El estudio muestra las reticencias entre tradiciones teóricas, experimentales y prácticas en la investigación científica.

Los científicos en la investigación básica, en este caso la biología molecular, sostienen que la ciencia no puede estar planificada por objetivos políticos. Dice uno de los entrevistados: «La investigación básica, en particular la biología celular, debe generar el conocimiento necesario antes de que pueda ocurrir algún avance real. La impaciencia, a pesar de que pueda estar justificada y ser comprensible por parte de los millones de pacientes de cáncer, no debería hacer 
que, bien las organizaciones científicas o los políticos, adopten medidas que, en último término, consuman grandes cantidades de dinero sin aportar un éxito real» (Hohlfeld 1982: 151). Esta forma de pensar reduce el problema del cáncer a acontecimientos clave en los procesos biológicos, que deben ser explicados por teorías moleculares. La consecuencia es que el progreso aquí sólo se valora en función de valores estrictamente epistémicos y la curación o no de los enfermos de cáncer dependería únicamente de los avances en la biología molecular.

El primer paso hacia la aplicación constituye el campo de «la investigación experimental del cáncer» que consiste en un tipo de investigación localizada detrás de la frontera de la «verdadera» ciencia, estructurada por las aún cuestiones teóricas fundamentales no resueltas. Este tipo de investigación está orientada no a la obtención de conocimiento por sí mismo sino que está dirigida a la solución de algunos problemas. Los científicos que trabajan en este campo comparten la idea con los de la investigación básica de que los problemas de salud de este tipo tienen que resolverse por instrumentos científicos sobre la base de la clarificación de los mecanismos biológicos subyacentes y que esto requiere «alta tecnología». También comparten con los de investigación básica que se necesitan expertos en el tema. Lo que les diferencia, fundamentalmente, es que los de la investigación experimental están motivados por el objetivo de que su trabajo revierta en beneficio de la humanidad, aunque también consideran relevante la explicación de los procesos estrictamente biológicos.

Cuando la motivación científica intrínseca combinada con una orientación a un fin externo a la ciencia ocurre en un campo con cuestiones teóricas clave aún no resueltas, entonces se da un tipo de investigación que corresponde a lo que entendemos por «investigación aplicada». Los científicos que realizan este tipo de investigación tratan de hacer llegar los resultados de la investigación a la clínica. Éste sería el caso de los investigadores del cáncer los cuales tienen las lealtades divididas entre investigación básica en biología y la orientación clínica. Un entrevistado señala que la denominación «Investigador del cáncer» es casi un invento. El investigador del cáncer está situado entre dos mundos. Estos son, por un lado, el mundo de la clínica, y, por el otro, la prestigiosa investigación básica, por ejemplo, la Sociedad Max Plack. La gente implicada en la investigación básica dirá que la investigación del cáncer no tiene una base sólida, que la preocupación por el cáncer como tema principal es una restricción para la investigación básica a gran escala (Hohlfeld 1982). Además, la formación de unos y otros es distinta y, por lo que Hohlfeld muestra en las entrevistas no parece fácil la colaboración entre científicos en investigación básica y aplicada. En primer lugar, los médicos piensan que no ha habido un avance real ni siquiera una contribución real al progreso médico en la lucha contra el cáncer desde la biología molecular. En segundo lugar, el modelo médico, teoréticamente, toma el modelo de las ciencias empíricas, física, química, etc. al mismo tiempo que 
descontextualiza el órgano y el individuo enfermo. En tercer lugar, a veces distintas especialidades médicas se disputan el tratamiento de los enfermos del cáncer. Finalmente, hay desconfianza de los médicos hacia los científicos en investigación básica, tal como muestra la afirmación de uno de los médicos entrevistados: «No podemos dejar a los enfermos en manos de los científicos.... No vamos a ser reducidos al papel de agentes de los científicos».

Dentro de este amplio campo de la investigación del cáncer están los estudios epidemiológicos. Se trata de estudiar qué es lo que enferma a la persona sana. Este no es el objetivo del médico que trata sólo a las personas ya enfermas. Las dificultades en este campo proceden, fundamentalmente, de las consecuencias políticas y sociales que pueden suponer los resultados de un estudio epidemiológico. La epidemiología puede considerarse también ciencia aplicada a partir de determinados factores y sus correspondientes enfermedades. En el caso del cáncer se trata de buscar indicadores de los que se ha encontrado una correlación con un tipo de tumor. Algunos de estos indicadores tendrán su base en la biología molecular pero otros pueden estar ligados a factores sociales en sentido amplio, desde determinadas conductas a factores medioambientales, pasando por tipos de dieta.

Hay una serie de preguntas que, en este momento, se plantean muchos científicos investigadores del cáncer: ¿por qué hay la sensación de que se ha avanzado más en la base teórica que en la técnica y organizativa?, ¿cuáles podrían ser los factores que hacen que, a pesar de conocerse muchos mecanismos del desarrollo de los tumores, el cáncer no puede curarse? Los casos de supervivencia (que en algunos cánceres han aumentado considerablemente en las últimas décadas) se deben más a técnicas que permiten detectar los tumores en fases iniciales que a verdaderas curaciones una vez se ha detectado la enfermedad. Podríamos decir que lo que falla aquí es el puente entre el conocimiento teórico y la necesidad que se trata de satisfacer.

Desde los años setenta en que Hohlfeld realizó el estudio han pasado tres décadas pero las cuestiones planteadas persisten y no se han resuelto. Desde determinados enfoques como el de «Ciencia-Técnica-Sociedad», con todas sus variantes, puede parecer que la frontera entre ciencia pura y aplicada se ha difuminado hasta no poder detectar sus límites. Hasta cierto punto es cierto si nos situamos a nivel práctico, pero no es lo mismo a nivel conceptual. Además, la convergencia en la práctica se debe, fundamentalmente, a la necesidad de justificar la financiación de la biología molecular a partir de los beneficios para el tratamiento del cáncer.

Frente a estas dificultades hay que preguntarse por las razones de las dificultades y tensiones en la investigación del cáncer. Una de ellas es el hecho de que concurren diversas ciencias, tanto puras como aplicadas, tales como la biología, la química, la medicina, la farmacología y las ciencias de la enfer- 
mería. Desde un análisis filosófico vamos a ver qué marcos metateóricos son especialmente adecuados para abordar este campo de investigación tan complejo y a la vez uno de los que tanta incidencia tiene en la vida de tantas personas a nivel planetario. ${ }^{3}$

\section{MARCOS TEÓRICOS}

Para dicho análisis vamos a tomar en consideración el modelo de ciencias de diseño de H. Simon (1969), la reflexión filosófica sobre el mismo de I. Niiniluoto (1993), y la praxiología como ciencia de la acción eficiente de T. Kotarbinski (1965), como marcos teóricos de una aproximación racionalista a la relación entre ciencia pura y aplicada.

\section{III.1. CIENCIAS DE DISEÑO}

Las ciencias de diseño son el resultado de un proceso de cientifización y mecanización de las artes en el sentido de habilidades, y de las actividades prácticas. H. Simon en The science of the artificial (1969) señala que el modelo tradicional de ciencia ofrece una imagen engañosa de campos como la ingeniería, medicina, arquitectura, economía, educación, etc. que están interesadas en el «diseño», en el sentido de objetivo, propósito, meta a conseguir, es decir, no tienen como objetivo saber cómo son las cosas sino cómo tienen que ser las cosas para conseguir determinados fines.

Niiniluoto toma la idea de las ciencias de diseño de Simon para abordar la ciencia aplicada y dar así una alternativa a la filosofía de la ciencia en el caso de que se trate de la aplicación de conocimientos científicos a la transformación de la realidad. Así distingue entre ciencias descriptivas (sería el tipo de ciencia que tradicionalmente se ha denominado «básica» o «pura») y las ciencias de diseño. En principio podríamos decir que las últimas son equivalentes a la ciencia aplicada, sin embargo, tanto para Simon como para Niiniluoto las ciencias de diseño tienen una entidad propia que no puede reducirse a la mera aplicación automática de los conocimientos científicos.

Histórica y tradicionalmente, la labor de las disciplinas científicas ha sido enseñar cómo son los fenómenos y cómo funcionan. La labor de las escuelas de ingeniería es enseñar sobre cosas artificiales: cómo construir artefactos que tengan las propiedades deseadas y cómo diseñarlas. Los ingenieros no son los únicos diseñadores profesionales. La actividad intelectual que produce artefactos materiales no es fundamentalmente distinta de la de prescribir remedios para un paciente, o la de programar un nuevo plan de ventas para una compañía o una política de asistencia social. El diseño, así construido, es el núcleo de la

3 Por supuesto no es el único pero posiblemente estos marcos teóricos serían igualmente adecuados para abordar otro tipo de problemas, desde el SIDA hasta los accidentes laborales. 
formación profesional; es el rasgo principal que distingue las profesiones de las ciencias.

Un punto a remarcar es que, a pesar de que el mismo Simon considera la medicina, el periodismo, la biblioteconomía, etc. como ciencias de diseño, el modelo de ciencias de diseño que la mayoría de autores toma para dotar de modelos teóricos a una ciencia de diseño son las ingenierías. También el progreso y otras cuestiones como su relación con las ciencias puras se abordan pensando en las ingenierías. Esto significa que hay una amplia labor por realizar, en el sentido de la adaptación de estos modelos de diseño (en un principio con la mirada puesta en las ingenierías) ${ }^{4}$ a otras disciplinas, y para el caso que nos ocupa a la medicina y a la investigación del cáncer.

\section{III.2. PRAXIOLOGÍA}

T. Kotarbinski (1962) define la praxiología como la ciencia de la acción eficiente, y su objetivo es investigar las condiciones de las que depende la maximización de la eficiencia. Partimos de que los enunciados de las ciencias de diseño son normas prácticas y, por tanto, sentencias praxiológicas, por ejemplo, «si quieres proteger a tu hijo de la varicela, vacúnalo». Así, el esquema general de una norma práctica es: «Bajo la circunstancia A es necesario (suficiente o aconsejable) hacer $\mathrm{B}$ a fin de conseguir $\mathrm{C}$.

En una norma práctica intervienen tres elementos: los fundamentos teóri$\cos$, la base técnica y la base conductual. Por fundamento teórico de una norma práctica entendemos cómo $\mathrm{C}$ depende causalmente de $\mathrm{B}$, y está en función del progreso cognoscitivo de la ciencia. En el caso de la vacuna los elementos químicos son la causa de la protección de la varicela. La base técnica consiste en los instrumentos, artefactos y técnicas específicas de lograr el fin propuesto, dependiendo del avance tecnológico que, a su vez, depende de aquellas disciplinas que más directamente están ligadas a los artefactos. En el caso de la vacuna puede haber distintas formas de darla, por ejemplo, por vía oral o inyectándola, con lo cual aún siendo los mismos componentes puede ser que una nueva forma de darla sea más efectiva o reporte menos efectos secundarios. La base conductual consiste en la selección y orden de las acciones que realizan los agentes a fin de alcanzar el fin propuesto de la forma más eficiente posible. En el caso de la varicela, podría haber cambios en la edad de poner la vacuna, cuántas veces hay que repetir la vacuna a lo largo de la infancia, etc.

Veamos cómo interpretar estos tres elementos en el caso de la investigación del cáncer. Toda la investigación en torno de los oncogenes constituiría la base teórica de la medicina del cáncer. Un ejemplo de avance a basado en el elemento

4 El papel de las ingenierías como disciplina de referencia para las ciencias de diseño es comparable al papel de la física en los modelos de ciencia de la Concepción Heredada. 
técnico lo tenemos en el desarrollo de un bisturí que detecta en segundos células cancerígenas. Según una noticia aparecida en El País (24-3-2000) científicos de los Laboratorios Nacionales Sandia del Departamento de Energía de Estados Unidos han desarrollado un bisturí destinado a detectar la presencia de células cancerígenas mientras el cirujano extirpa un tumor oscurecido por la sangre, el músculo y la grasa. El aparato se llama «láser de microcavidades biológicas» y ha logrado distinguir en el laboratorio entre células cultivadas, consistentes en células normales del cerebro llamadas astrocitos, y su forma maligna, llamada «glioblastomas». Esto puede ayudar a los cirujanos a eliminar con precisión los crecimientos malignos a la vez que reduce al mínimo la cantidad de tejido sano que se extirpa. En cuanto a la base conductual, en estos momentos de circulación del conocimiento a nivel planetario (al menos como posibilidad) las acciones a seguir responden a diferencias en las políticas sanitarias, en función de factores económicos, sociales y políticos, lo cual, indudablemente, implican diferencias en las expectativas de vida para los enfermos de cáncer.

\section{LA DINÁMICA DE LAS CIENCIAS DE DISEÑO}

La dinámica científica fue abordada por el enfoque historicista, teniendo como referencia indiscutible la obra de T. Kuhn sobre la estructura de las revoluciones científicas, al que le siguieron I. Lakatos, N. R. Hanson, L. Laudan, entre otros muchos. Nunca se especificó si se trataba de ciencias puras o de diseño, sin embargo todos los ejemplos históricos a los que los filósofos han aludido siempre han sido de ciencias puras o descriptivas. Sin embargo, desde la filosofía de la tecnología sí hubo análisis sobre el cambio tecnológico, centrándose, fundamentalmente, en la invención (conjunción de diversos elementos técnicos que tienen como resultado una nueva tecnología) y la innovación (aplicación de tecnología a un nuevo ámbito). En este sentido, también se ha tenido en cuenta la adopción y aceptación por parte del usuario de los cambios tecnológicos. Un ejemplo de ello es el estudio de D. Wojick (1979) sobre las revoluciones tecnológicas, centradas, bien en el acto de invención bien en el impacto social de la nueva tecnología. El factor humano se aborda no por las consecuencias de la tecnología para la sociedad sino por el grado de aceptación de las innovaciones (revoluciones) por la comunidad profesional (ingenieril, médica, educativa, etc.). Esta idea nos retrotrae a Kuhn, que pone el énfasis en los libros de texto como una forma de aceptación del nuevo paradigma, lo cual incide en la misma idea de Wojick de la importancia de la aceptación de las revoluciones tecnológicas por la comunidad profesional.

Ahora bien, si tenemos en cuenta la praxiología, para la que el elemento técnico es sólo uno de los elementos de las normas prácticas, lo que no podemos hacer es identificar revoluciones en las ciencias de diseño con revoluciones tecnológicas. Además, no todas las ciencias de diseño giran en torno a la tec- 
nología, tal es el caso de la biblioteconomía o las ciencias de la educación, a no ser que trivialicemos la idea de tecnología y consideremos que la utilización del ordenador es ya suficiente para catalogar la ciencia como tecnológica. Incluso en el caso de la investigación del cáncer, en que las técnicas son capitales para el diagnóstico, no la consideraríamos una disciplina tecnológica.

Una de las cuestiones que no pueden soslayarse a la hora de abordar los cambios científicos es el proceso de evaluación que es el núcleo del razonamiento práctico. En el proceso de evaluación intervienen valores y hechos. El análisis de coste-beneficio es un ejemplo paradigmático de este tipo de evaluación práctica; sin embargo, el valor neto de una solución no se reduce a su valor monetario ya que hay factores sociales, éticos, etc., así como consideraciones de utilidad, eficiencia y seguridad que hay que tener en cuenta en un análisis de coste-beneficio. Los sistemas de evaluación articulados en procedimientos estándar juegan un papel central en la práctica tecnológica, y definen los principios normativos que rigen en la solución de problemas sociales. Sin embargo, los sistemas de evaluación establecidos pueden ser incorrectos. Podemos descubrir que nuestras prácticas y las normas por las que se rigen dejan fuera factores que deberían tenerse en consideración o que no se les da el peso que deberían tener. Esto plantea la cuestión de cómo pueden cambiar los sistemas de evaluación. Para analizar estos cambios Wojick recurre a Kuhn con el objetivo de que lo que Kuhn dice de las revoluciones científicas pueda aplicarse a los cambios en las prácticas tecnológicas, en el sentido de que el sistema de evaluación aceptado juega un papel en la organización del uso de tecnología análogo al papel que juegan el paradigma científico en la organización de la explicación científica.

El objetivo de un sistema de evaluación es guiarnos para trasformar la actual situación en otra que sea la mejor posible. Aquí lo que el valor representa para un sistema de evaluación equivale a lo que la verdad es para la ciencia. Las anomalías surgen cuando el sistema de evaluación no nos lleva al mejor estado posible. Del mismo modo que el fracaso de un paradigma para dar una explicación de un fenómeno provoca una anomalía para el paradigma, el fracaso repetido de unos procedimientos estándar para eliminar determinadas enfermedades provoca una anomalía para un sistema de evaluación aceptado hasta el momento.

Otro tipo de anomalía surge cuando un nuevo conocimiento científico y técnico nos permite ver que nuestros procedimientos no evalúan correctamente determinados factores. Ejemplos de este tipo de anomalía los encontramos en el descubrimiento, como resultado de los avances de la bioquímica, de los efectos, a largo término, de los pesticidas. $\mathrm{O}$ el descubrimiento de los efectos secundarios de los aditivos en los alimentos a través de los avances de la medicina, y el descubrimiento de los efectos ecológicos adversos de los pantanos a través de los avances de la ecología. Este tipo de anomalía puede ser problemática de 
detectar porque el conocimiento que provoca la anomalía puede estar en otro campo disciplinar. Por ejemplo, hasta mediados de los sesenta los ingenieros no estuvieron en contacto con los resultados de la ecología, con lo cual mal podían percatarse de la anomalía ya que desconocía la información necesaria para detectarla. Además, del mismo modo que los paradigmas son inconmensurables, también podemos encontrar sistemas de valoración que partan de puntos de vista inconmensurables.

Está claro que la aportación de Wojick es importante e innovadora para esclarecer el estudio de los cambios en la ciencia aplicada, aunque los ejemplos que toma proceden, mayormente, de la ingeniería en todas sus ramas. Pero las cuestiones que plantea son aplicables a la medicina del cáncer. Pensemos en la evaluación de las distintas terapias, de los cambios de tratamiento y un sinfín de situaciones frente a las que hay que tomar una serie de decisiones a partir de criterios científicos, éticos, sociales, económicos, estéticos, etc. Todo ello supone sistemas de evaluación que han ido cambiando con el tiempo a partir de nuevos conocimientos, tecnologías nuevas y aceptación de valores más allá de los estrictamente científicos.

\section{El PROGRESO EN LA INVESTIGACIÓN DEL CÁNCER}

El progreso en las disciplinas prácticas depende de los tres elementos que integran la praxiología, a saber: base teórica, técnica y organizativa. Vamos a ver cómo se concretaría en el caso de la investigación del cáncer, partiendo de que el objetivo es curar la enfermedad o al menos paliar los efectos y obtener una mejor calidad de vida. Las ciencias de diseño implicadas en el tratamiento de la enfermedad del cáncer son la medicina, la farmacología y las ciencias de la enfermería. Se trata del diseño de fármacos, de radioterapia, de quimioterapia y de cirugía. Tanto respecto a las ciencias de diseño implicadas como en los distintos elementos que intervienen no se trata de una enumeración exhaustiva sino de lo más representativo.

El progreso en base teórica consiste en los conocimientos aportados por la biología molecular (muy especialmente el funcionamiento de la célula), la química (en los tratamientos de quimioterapia) y la física (para la radioterapia). El progreso en la base técnica viene dado por los avances en las técnicas de escáner, los aparatos para realizar análisis y las técnicas de medicina nuclear, resonancia magnética, etc. Y en la base organizativa y conductual el progreso está relacionado con la organización del sistema sanitario, las nuevas formas de cirugía y los modos de acción respecto a los enfermos. No cabe duda de que cualquier avance en uno de los elementos supondrá, a la larga, cambios positivos en los otros elementos, pero pueden darse de forma independiente y no necesariamente coincidentes en el tiempo. Por ejemplo, con los mismos conocimientos teóricos, pueden darse avances importantes en la curación de la enfermedad 
con nuevas técnicas de detección de tumores. Respecto a los cambios en la base conductual, las diferencias en la supervivencia de los enfermos de cánceres son más que evidentes. Sólo hace falta comparar los índices de supervivencia en países como África y en Europa.

A partir de estas consideraciones podemos decir que en las ciencias descriptivas cuando comparamos dos teorías nos referimos a que una teoría T1 es mejor que otra teoría T2, porque explica mejor determinados fenómenos, tiene más evidencia empírica, tiene modelos matemáticos más potentes, etc. La cuestión está en cuál sería el equivalente para las ciencias de diseño. En principio podríamos decir que el diseño D1 es mejor que el diseño D2. Pero la siguiente pregunta sería en qué sentido es mejor, a lo cual podemos responder que D1 cubre mejor que D2 las necesidades reconocidas o que D1 cumple mejor que D2 las funciones atribuidas. Finalmente, nos preguntamos cuáles son los factores que hacen que D1 cubra mejor las necesidades que D2, lo cual nos remite a los tres elementos de la praxiología, cuya convergencia contribuirá a la eficiencia en la consecución del fin, en este caso a la curación de los enfermos de cáncer.

\section{CONCLUSIONES}

Si tenemos en el punto de mira al enfermo de cáncer, la investigación sobre dicha enfermedad sólo puede tener una perspectiva interdisciplinar, multidisciplinar y transdisciplinar. ${ }^{5}$ La idea es que la eficiencia en la consecución de los objetivos está en función de la colaboración de todas las disciplinas implicadas en el tratamiento del cáncer.

La perspectiva interdisciplinar la distingo de enfoques holistas que impliquen el cuestionamiento de estudios especializados y que solamente valoren las aproximaciones globales. A la hora de tomar decisiones o de valorar el progreso no cabe duda que hay que tener en cuenta los diferentes factores que inciden en el tratamiento de la enfermedad, fruto de las aportaciones de las diferentes disciplinas implicadas en el campo de la investigación del cáncer.

\section{REFERENCIAS BIBLIOGRÁFICAS}

BERENBLUM, I. 1967: Cancer research today. Oxford: Pergamon. BÖHME, G.W., R. VAN DEN DAELE, W. HOHLFELD, W. KROHN \& W. SCHÄFER, 1983: Finalization in science. The social orientation of scientific progress. Dordrecht (Holland): D. Reidel.

5 No voy a entrar en el análisis de las diferencias entre estos tres conceptos, no es necesario para el propósito de este trabajo. Es suficiente la idea de que para abordar la enfermedad del cáncer es necesario la intervención de varios campos disciplinarios. 
CASACUBERTA, D. y ESTANY, A. 2003: ¿EUREKA? El trasfondo de un descubrimiento sobre el cáncer y la genética molecular. Barcelona: Tusquets.

GASPARSKI, W.W. \& T. PSZCZOLOWSKI (eds.), 1983: Praxiological studies. Polish contributions to the science of efficient action. Boston: Reidel.

GROSS, L. 1970: Oncogenic viruses, Pergamon, Oxford.

HOHLFELD, R. 1982: «Two scientific establishments which shape the pattern of cancer research in Germany: basic science and medicine», en Elias, N., Martins, H., y Whitley, R. (eds.) Scientific establishments and hierarchies. Sociology of Sciences, v. VI: 145-168, Dordrecht (Holland), D. Reidel.

HOHLFELD, R. 1983: «Cancer research. A study of praxis-related theoretical developments in chemistry, the biosciences and medicine», En Böhme, G. Van Den Daele,W. Hohlfeld, R., Krohn,W. y Schäfer,W., The finalization of science, Dordrecht (Holland), D. Reidel.

KOTARBINSKI, T. 1965: Praxiology. An introduction to the science of efficient action. New York: Pergamon Press.

KRANZBERG, M. 1967: «The unity of science-technology», American Scientist, v. 55, n. 1, pp. 48-66.

KRANZBERG, M. 1968: «The disunity of science-technology», American Scientist, v. 56, n.1, pp. 21-34.

NIINILUOTO, I. 1993: «The aim and structure of applied research», Erkenntnis, v. 38, pp.1-21.

POTTER, R. 1964: «Biochemical perspectives in cancer research», Cancer Research, v. 24, pp. 1085-1098.

PROCTOR, R.N. 1995: Cancer wars. How politics shapes what we know and do not know about cancer, Basic Books, New York.

SIMON, H. 1996: The science of the artificial, The MIT, Cambridge (MASS), $\left(3^{\mathrm{a}}\right.$ edición).

WEINBERG, R.A. 1998: Racing to the begining of the road: The search for the origin of cancer, W.H. Freeman,.

WOJICK, D. 1979: «Philosophy of technology and the structure of technological revolutions». En G. Bugliarello y D.B. Doner (eds.) The history and philosophy of technology, University of Illinois Press, Chanpaign (ILL). 
Biología aplicada...

Anna Estany es Catedrática de Lógica y Filosofía de la Ciencia en el Departamento de Filosofía de la Universidad Autónoma De Barcelona

Líneas de investigación:

Filosofía de las ciencias de diseño. Enfoque cognitivo en la filosofía de la ciencia.

Publicaciones recientes:

ESTANY,A.\& D. CASACUBERTA 2012: «Contributions of Socially Distributed Cognition to Social Epistemology: The Case of Testimony». EIDOS, No 16, pp: 40-68.

ESTANY, A. 2012: «The Stabilizing Role of Material Structure in Scientific Practice». Philosophy Study, v.2, nº 6, pp: 398-410.

Dirección electrónica: Anna.estany@uab.cat 